\title{
Towards unusual mechanical properties of tensegrity lattice metamaterial
}

\author{
Anna Al Sabouni-Zawadzka, and Wojciech Gilewski ${ }^{1}$ \\ Warsaw University of Technology, Faculty of Civil Engineering, Division of Timber Structures, \\ ul. Lecha Kaczyńskiego 16, Warsaw 00-637, Poland
}

\begin{abstract}
In the present paper a novel cellular metamaterial based on a tensegrity pattern is presented. The material is constructed from supercells, each of which consists of eight 4-strut simplex modules. The proposed metamaterial exhibits some unusual properties. It is possible to control its mechanical characteristics by adjusting the level of self-stress or by changing the properties of structural members. A discrete model is introduced to identify the properties of the considered metamaterial and to estimate how the applied self-stress and the characteristics of cables and struts affect the whole structure. The performed analyses is an attempt to the future design of tensegrity lattices which can be recognised as a metamaterial for engineering applications.
\end{abstract}

\section{Introduction}

Metamaterials are usually defined as man-designed and man-made, not observed in nature, composite structures with unusual or non-typical properties [1-3]. Features of metamaterials are determined mainly by morphology of the structure in the scale bigger than molecular, and in smaller degree by chemical or phase composition. This area has been under considerable and important scientific research in recent years. Many state-of the-art applications refer to electromagnetic waves and phenomena [4-6], solar photovoltaic cells and panels [7,8], energy absorption, including seismic [9] and acoustic waves [10,11] and mechanical metamaterials [12-16] (for example with unusual dynamic properties, negative Poisson's ratio, non-typical modulus of extension and volumetric changes, ultra-light and ultra-stiff materials). In the context of various definitions [17], the metamaterial is more close the term smart structure then smart material.

Standard engineering materials when compressed along a particular direction are most commonly observed to expand in the directions orthogonal to the applied load. This property is measured by a Poisson's ratio, which is a good example to characterize the mechanical metamaterial. Positive Poisson's ratio in the range of 0.0 to 0.5 is observed for the majority of engineering materials, which means it is a typical material property. However, theory of elasticity permits negative values and for anisotropic materials also the

${ }^{1}$ Corresponding author: w.gilewski@il.pw.edu.pl 
coefficients bigger then 0.5. Negative Poisson's ratios are not observed in nature and this is why metamaterials with such a property are being looked for.

An interesting type of structures which allow to build materials with negative Poisson's ratios are tensegrities $[18,19]$. For the purpose of this paper, a tensegrity structure is defined as a pin-jointed system with a particular configuration of cables and struts that form a statically indeterminate structure in a stable equilibrium. Tensegrities consist of a discontinuous set of compressed elements inside a continuous net of tensioned members, which have no compressive stiffness. To major advantages of tensegrity systems belong: large stiffness-to-mass ratio, deployability, reliability and controllability [17].

The properties of tensegrity metamaterials within the continuum mechanics model were recently analyzed in [20]. The objective of the present paper is to develop a metamaterial based on the 4-strut simplex tensegrity module within discrete model. Its mechanical characteristics can be controlled with the self-stress state and cable to strut properties ratio, including positive or negative values of Poisson's ratio.

\section{Tensegrity lattice}

The aim of this paper is study behaviour of structure stabilized by cables tension. To do this infinitesimal mechanisms and self-stress states should be determined and next the influence of the level of self-stress on displacements should be analysed. Identification infinitesimal mechanisms and a self-stresses in structures is possible by using the second order theory. In order to take account the effect of additional compression the third order theory should be used.

\subsection{Unit cell and metamaterial}

The proposed metamaterial is based on one of the best known tensegrity modules - a 4-strut simplex (Figure 1). As all typical tensegrity structures, it is a pin-jointed system consisting of isolated compressed elements (four struts) inside a continuous net of tensioned members (twelve cables) [18-19]. The 4-strut simplex module is obtained from a regular prism by rotating one of its bases 135 degrees clockwise or counter clockwise.

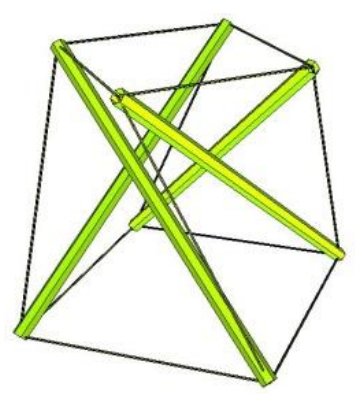

Fig. 1. Geometry of a unit cell - a 4-strut simplex module.

One of the unique features of tensegrities are infinitesimal mechanisms that are balanced with self-stress states [18-19]. The considered simplex module has one 
infinitesimal mechanism (Figure 2a) and one corresponding self-stress state (Figure 2b) self-stress is expressed by the relative forces in struts and cables with a multiplier $\mathrm{S}_{0}$.

a)

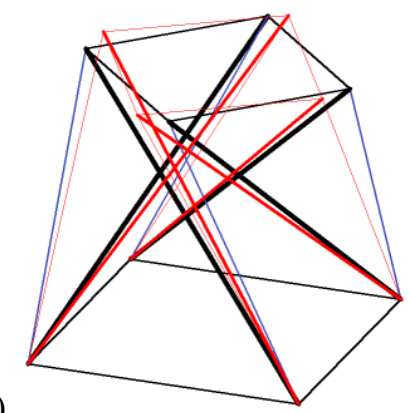

b)

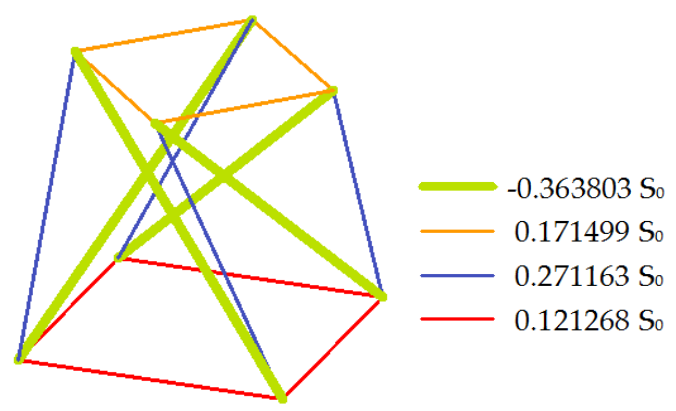

Fig. 2. 4-strut simplex: (a) Infinitesimal mechanism; (b) Self-stress state.

Simplex tensegrity modules described above can be arranged in different patterns to form a material with certain properties. Depending on the type of the module used (with the basis rotated clockwise or counter clockwise) and the way in which the modules are connected, a material with different mechanical characteristics can be obtained. In the present paper a material with orthotropic properties is proposed, as it exhibits some special features such as a zero or even negative Poisson's ratio.

Following this reasoning, an eight-module supercell (Figure 3a), built from two fourmodule layers, was considered. The upper layer of the system was created by putting the four-module layer upside-down and connecting it with the bottom layer through common cables. Metamaterial based on the proposed sequence of modules is presented in Figure 3b.

a)

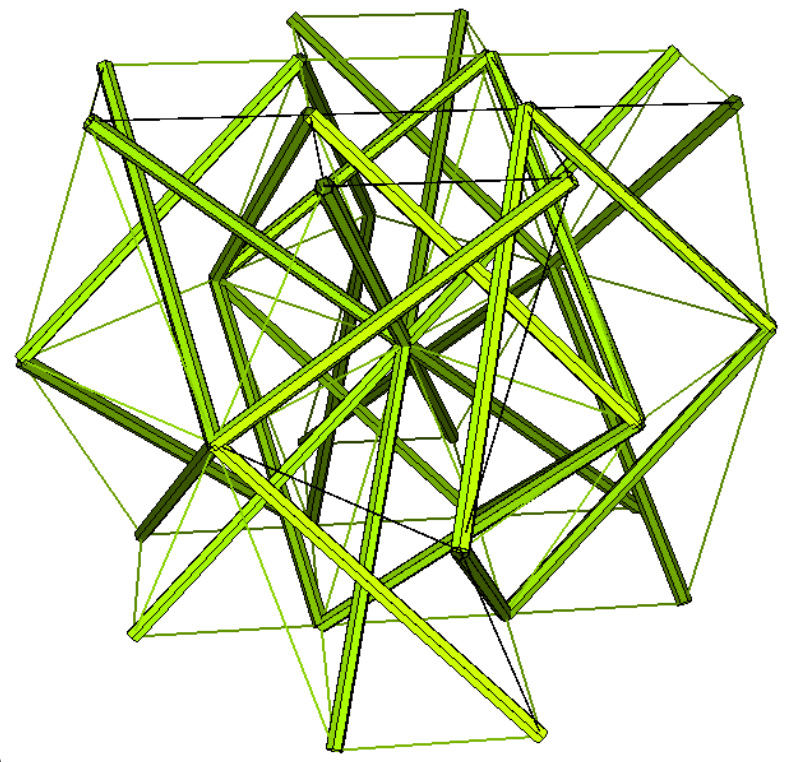


b)

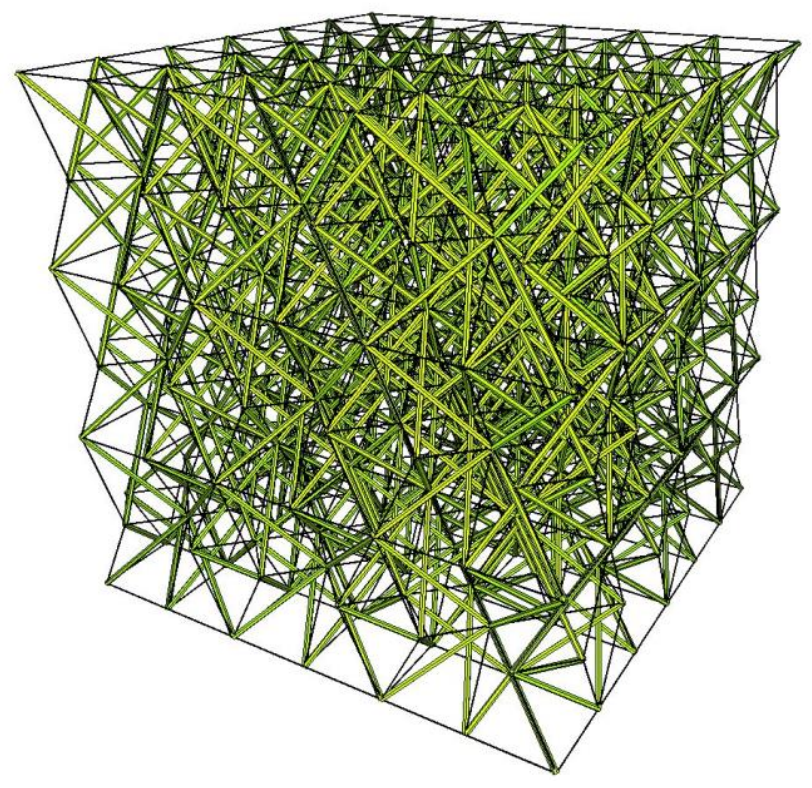

Fig. 3. Geometry of an eight-module supercell (a) and metamaterial (b).

\subsection{Mathematical formulation - finite element method}

The equilibrium equation for geometrically nonlinear model (third order theory) is presented in the form:

$$
\left(\mathbf{K}_{L}+\mathbf{K}_{N L}^{I}+\mathbf{K}_{N L}^{I I}\right) \mathbf{q}=\mathbf{P}
$$

where $\mathbf{q}$ is displacement vector and $\mathbf{P}$ is the load vector, $\mathbf{K}_{L}$ is the linear stiffness matrix and $\mathbf{K}_{N L}^{I}$ is the pre-stress stiffness matrix called the geometric stiffness matrix and $\mathbf{K}_{N L}^{I I}$ is the initial strain matrix $[21,22]$. For truss structures the linear stiffness matrix can be write as:

$$
\mathbf{K}_{L}=\mathbf{B}^{T} \mathbf{E} \mathbf{B}
$$

where $\mathbf{B}$ is the compatibility matrix and $\mathbf{E}$ is the elasticity matrix [23].

As it is developed in [24] the geometric stiffness matrix can be also expressed in algebraic form:

$$
\mathbf{K}_{N L}^{I}=\mathbf{C}^{T} \mathbf{S C}
$$

where $\mathbf{C}$ is the rotation matrix and $\mathbf{S}$ is a diagonal matrix of self-stress forces.

Based on singular value decomposition of compatibility matrix $\mathbf{B}$ infinitesimal mechanisms and self-stress states are identified [25]. Next the pre-stress stiffness matrix depended on the self-stress state is calculated and spectral analysis taking into account this matrix is used. Geometric non-linear analysis, including the initial strain matrix, is made using the Sofistik software. 


\section{Results of the analysis}

The eight-module supercell is analysed as an introduction to the study of mechanical properties of the proposed metamaterial. The supercell of the dimensions $4 a \times 4 a(a=1 \mathrm{~m})$ is expanded by an uniformly distributed pressure $q=2.5 \mathrm{kN} / \mathrm{m}^{2}$ transformed to the nodes (with the forces $P=0.5 q a^{2}$ ) as it is shown in Figure 4. Boundary conditions are applied to avoid rigid body motions. The following data are selected: strut cross-section $A_{s}=0.0726 \mathrm{~m}^{2}$, strut and cable Young modulus $E_{s}=600 \mathrm{GPa}$. The parametric study can be done for the ceble to strut ratio $k=E_{c} A_{c} / E_{s} A_{s}$ and the self-stress ratio $\sigma=S_{0} / E_{s} A_{s}$.

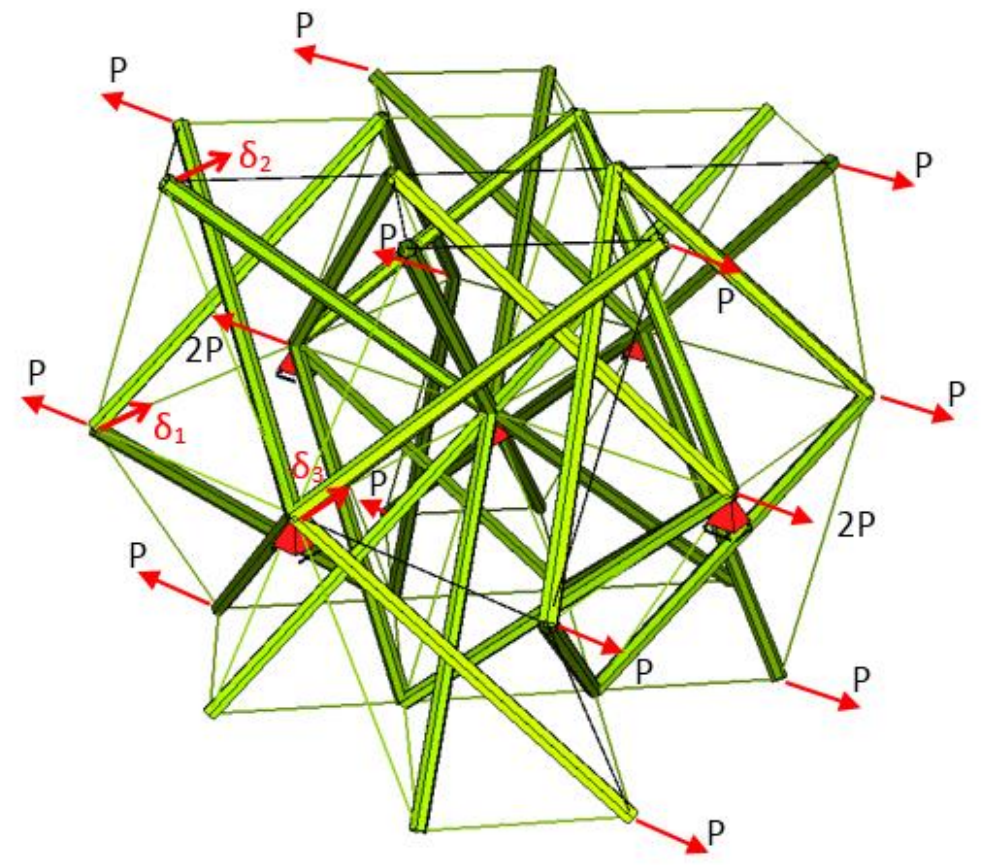

Fig. 4. Tensegrity metamaterial with load (P), boundary conditions and selected displacements $\delta_{1}, \delta_{2}$ ,$\delta_{3}$.

The results presented below are selected to show the influence of the parameter $k$ for the level of displacements in the direction perpendicular to the pressure applied (displacements $\delta_{i}, i=1,2,3$ in Figure 4). Second- and third-order theory provide to nearly identical results for the selected sequence of the modules.

An example of results obtained for the parameters $\sigma=0.1, k=0.1$ are presented in Figure 5 . Figure 6 gives a comparison of three displacements on the planes perpendicular to load direction. The values decrease if the parameter $k$ increase (if the cables are more stiff). The most interesting is the curve for the displacement $\delta_{3}$ in the middle of the wall. The value is positive for small $k$, then is close to zero and finally is negative. It means that negative value of the Poisson's ratio can be designed for the analysed metamaterial. 
)
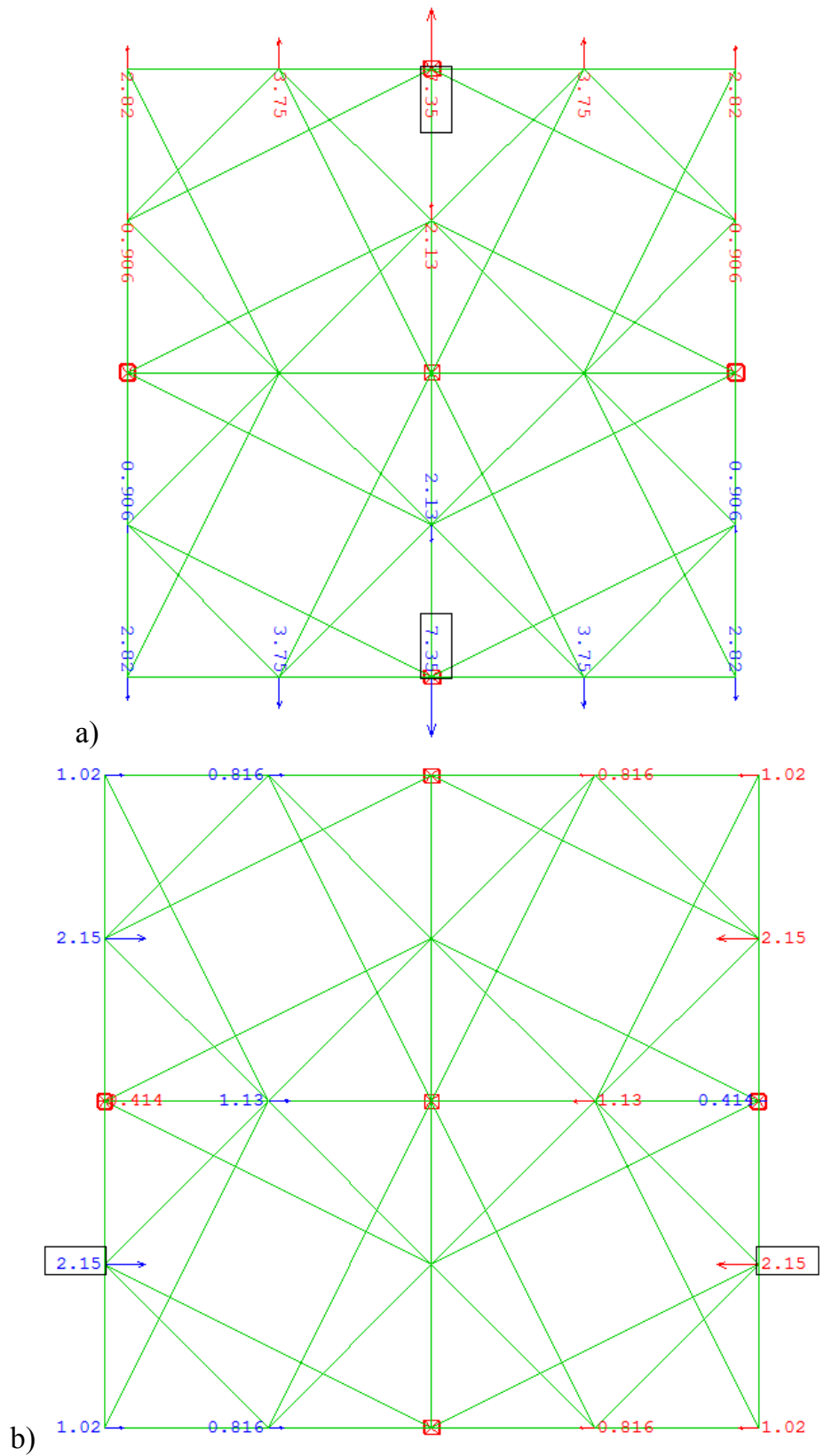

Fig. 5. Horizontal displacements in two directions: following the load (a), perpendicular the load (b), for $\sigma=0.1, k=0.1$. 


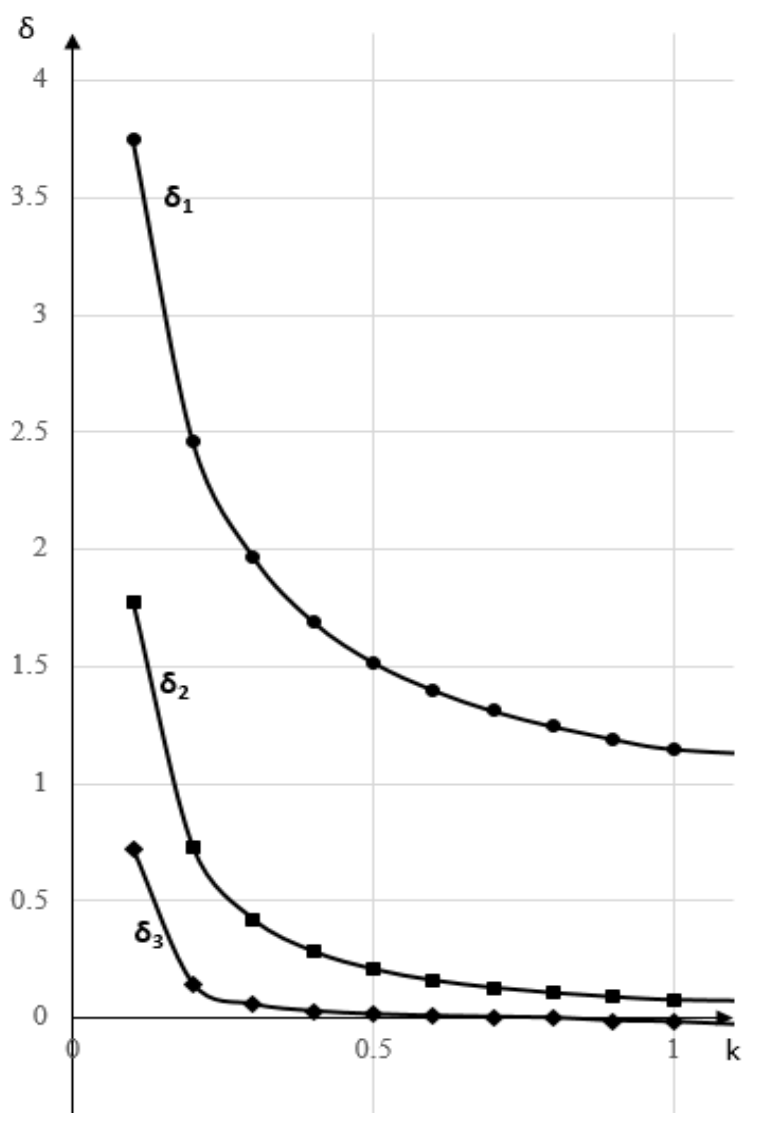

Fig. 6. Relation between selected displacements and the stiffness of cable to the stiffness of struts ratio (k) - obtained for $E_{s} A_{s} / q a^{3}=10000$.

\section{Conclusions}

The present paper focuses on the development and analysis of a novel cellular metamaterial based on the simplex tensegrity pattern. The proposed material is constructed from supercells, each of which consists of eight 4-strut simplex modules. Using a discrete model an eight-module supercell is analysed.

The proposed unit cell is an anisotropic structure. However, it can be arranged in such a way that that the material based on the simplex tensegrity pattern exhibits orthotropic properties. Such a material is considered in this paper. The developed metamaterial has some unusual properties, which are typical for smart structures. It is possible to control its mechanical characteristics by adjusting the level of self-stress or by changing the properties of structural members.

One of the most important features of the proposed cellular metamaterial is a unique behaviour of some of its Poisson's ratios. Depending on the applied control parameters they can be positive, zero or even negative. It is possible due to the occurrence of infinitesimal mechanisms balanced with self-stress states. 


\section{References}

1. T.J. Cui, D.R. Smith, R. Liu, Metamaterials. Theory, Design and Applications, Springer, New York, Dordrecht, Heidelberg, London, (2010)

2. N. Engheta, R.W. Ziolkowski, Metamaterials. Physics and Engineering Explorations. Wiley-Interscience, New York, (2006)

3. G. Singh, Ranji, A.A. Marwaha, Review of Metamaterials and its Applications, International Journal of Engineering Trends and Technology, 19 (6), pp. 305-310, (2015)

4. D. Schurig, J.J. Mock, B.J. Justice, S.A. Cummer, J.B. Pendry, A.F. Starr, D.R. Smith, Metamaterial electromagnetic cloak at microwave frequencies. Science, 314, pp. $977-$ 980, (2006)

5. C.M. Soukoulis, S. Linden, M. Wegener, Negative refractive index at optical wavelengths. Science, 315, pp. 47-49, (2007)

6. C.M. Soukoulis, M. Wegener, Past achievements and future challenges in the development of three-dimensional photonic metamaterials. National Photonics, 5, pp.523-530, (2011)

7. A. Vora, J. Gwamuri, N. Pala, A. Kulkarni, J.M. Pearce, D.O. Guney, Exchenging ohmic losses in metamaterial absorbers with useful optical absorption for photovoltaics. Science Report, 4, 4901, (2014)

8. C. Wu, B. Neuner III, J. John, A. Milder, B. Zollars, S. Savoy, G. Shvets, Metamaterial-based integrated plasmonic absorber/emitter for solar thermosphotovoltaic systems. Journal of Optics, 14, 024005, (2012)

9. S. Brule, E.H. Javelaud, S. Enoch, S. Guenneau, Experiments on seismic metamaterials: molding surface waves. Physical Review Letters, 112, 133901-1-5, (2014)

10. H. Chen, C.T. Chan, Acoustic cloaking in three dimensions using acoustic metamaterials. Applied Pgysics Letters, 91, 183518, (2007)

11. J. Mei, G. Ma, M. Yang, Z. Yang, W. Wen, P. Sheng, Dark acoustic metamaterials as super absorbers for low-frequency sound. Nature Communications, 3, 756, (2012)

12. J.B. Lee, S. Peng, D. Yang, Y.H. Roh, H. Funabashi, N. Park, E.J. Rice, L. Chen, R. Long, M. Wu, D. Luo, A mechanical metamaterial made from a DNA hydrogel. Nature Nanotechnology, 7, pp. 816-820, (2012)

13. K. Bertoldi, P.M. Reis, S. Willshaw, T. Mullin, Negative Poisson's ratio behavior by an elastic instability. Advanced Materials, 22, pp. 361-366, (2010)

14. M. Kadic, T. Buckmann, N. Stenger, M. Thiel, On the practicability of pentamode mechanical metamaterials. Applied Physics Letters, 100, 191901 (2012)

15. J.H. Lee, J.P. Singer, E.L. Thomas, Micro-/Nanostructured mechanical metamaterials. Advanced Materials, 24, pp. 4782-4810, (2012)

16. X. Zheng, H. Lee, T.H. Weisgraber, M. Shusteff, J. Deotte, E.B. Duoss, J.D. Kuntz, M.M. Biener, Q. Ge, J.A. Jackson, S.O. Kucheyev, N.X. Fang, C.M. Spadaccini, Ultra-light, ultra-stiff mechanical metamaterials. Science, 344, pp. 1373-1377, (2014)

17. W. Gilewski, A. Al Sabouni-Zawadzka, On possible applications of smart structures controlled by self-stress. Archives of Civil and Mechanical Engineering, 15, pp. 469478, (2015) 
18. R.E. Skelton, M.V. de Oliveira, Tensegrity Systems, Springer, Dordrecht, Heidelberg, London, New York, (2009)

19. R. Motro, Tensegrity: Structural Systems for the Future, Kogan Page Science, London, (2003)

20. A. Al Sabouni-Zawadzka, W. Gilewski, Smart metamaterial based on the simplex tensegrity pattern. Materials, 11, 637, pp. 1-14, (2018)

21. O.C. Zienkiewicz, R.L. Taylor, The finite element method. Vol. 1. The basis. Butterworth-Heinemann, New Jersey, (2000)

22. SOFISTIK Documentation, (2017)

23. T. Lewiński, On algebraic equations of elastic trusses, frames and grillages. Journ. Theoret. Appl. Mech., 39, pp. 307-322, (2001)

24. J. Pełczyński, W. Gilewski, An extension of algebraic equations of elastic trusses with self-equilibrium system of forces. $6^{\text {th }}$ European Conference on Computational Methods, 11-15 June 2018, Glasgow, UK

25. W. Gilewski, J, Kłosowska, P. Obara, Application of singular value decomposition to the qualitative analysis of trusses and tensegrity structures [in Polish], Acta Sci. Pol. Architectura, 14, pp.3-20, (2015) 